\title{
D'un monde boiteux au bonheur retrouvé : abolition des contradictions dans quelques romans de Françoise Lalande
}

\author{
Martine Renouprez \\ Université de Cadix
}

\section{Introduction}

Les récits de Françoise Lalande sont des scènes où s'affrontent tous les antagonismes, de l'actualité politique au cœur de l'intime - familial ou intérieur -. Cette guerre permanente que l'homme mène contre lui-même et contre autrui, Lalande l'interroge et l'analyse à travers ses personnages, tentant de mesurer comment résoudre ces conflits qui les minent pour atteindre des sentiments de joie et de sérénité. Trois romans significatifs se succèdent dans notre approche de l'œuvre: le tout premier, Le Gardien d'abalones, écrit en 1983, met en scène une seule protagoniste, Louise Keil, aux prises avec ses démons - ses propres contradictions -, et qui fuit un acte qu'elle a commis et qui la met en porte à faux vis-à-vis d'un monde qui l'engloutit. Le second roman, La Séduction des hommes tristes (2010), confronte, dans un paysage identique au premier roman, deux êtres que tout oppose, mais qui vont trouver l'unité dans l'amour et, au même instant, dans la mort. Enfin, Nous veillerons ensemble sur le sommeil des hommes (2012) montre l'unité rendue possible et atteinte par trois personnages, Léa, Lila et Julius Keil, dans l'amour et de la mort, symboles de toutes les contradictions - et y en a-t-il de plus insolubles que celles-là? C'est un au-delà de la pensée rationnelle que nous apportent ces romans; le dualisme qui découle des principes logiques du rationalisme ravage un monde en proie aux conflits et qui ne trouve pas de solution aux antagonismes qu'il provoque. L'évolution de cette question $\mathrm{du}$ premier au dernier roman de Françoise Lalande trace des voies lumineuses pour sortir des apories du monde contemporain.

\section{Le Gardien d'abalones : la passion à l'ombre du crime}

Ce roman pose la question de nombreuses contradictions qui laissent perplexe. Comment peut-on à la fois être coupable et innocent? Comment peut-on à la fois vouloir et ne pas vouloir? Y a-t-il des crimes nécessaires? Le décalage découlant de ces paradoxes est une constante du récit. Les réalités extérieure et intérieure offrent des combats entre des entités qui ne s'accordent pas, mais s'affrontent sous l'apparence d'une harmonie trompeuse. Le monde est boiteux. Nous sommes au Mexique, à Taxco, le 24 décembre, de midi à minuit : des femmes âgées profitent de leur fortune pour jouir de jeunes garçons démunis : pas d'amour dans ces rencontres avec «ces hommes aux yeux remplis de rage» (Lalande 1994, 20). Le monde 
extérieur ne s'accorde pas non plus avec Louise Keil, une protagoniste décalée de la réalité, qui refuse ce qui va de soi, et s'efforce d'éviter toute position confortable qui pourrait l'endormir : elle commande à boire ce qu'elle déteste : " une eau gazeuse » (23); elle se lève et marche bien que ses pieds «la fassent souffrir» (Id.), car il n'est pas question «de s'installer quelque part» (22). Ce sont ces paradoxes intimes qui maintiennent en alerte sa conscience vive; c'est la contradiction qui est recherchée dans ses tensions, à rebours de l'aspiration au bonheur qui hante l'humanité. La dureté, assimilée à une boule dans la gorge, va chez elle de pair avec la joie : « Parfois, de bonheur, elle avait l'impression que l'air allait lui manquer » (61); « elle ne voulait pas être heureuse » (100). Sans doute Louise Keil ne s'aime-t-elle pas.

Le doute hante sa conscience coupable, car elle est convaincue d'avoir commis un meurtre pour lequel elle craint de se faire arrêter à tout moment. Le lecteur n'est pas tout de suite informé de la nature du délit, mais Louise est d'emblée désignée comme une femme criminelle. Et l'accent est mis sur les conséquences qui en découlent, dont l'isolement: "Être en dehors. Hors de. Ni au-dessus. Ni en dessous. Mais loin de » (15). Son acte l'a éloignée du reste de l'humanité... « une solitude aussi grande » (27). Cette place singulière, qui la situe hors du commun, est issue de sa volonté; c'est sciemment qu'elle se distingue et se met à l'écart : « elle voudra être "hors des autres" » (22). Cette solitude la renvoie à elle-même, en une annulation de soi tout autant recherchée : «Louise Keil ne cherche-t-elle pas son propre anéantissement? » $(19,39)$. Pourtant, «l'anéantissement dont elle se croit avide n'est pas la mort» (54). Cet éloignement, cet isolement des êtres et des choses ne la paralysent pas. Au contraire, Louise active une conscience qui ne cesse de s'interroger. Et cette interrogation, la conscience d'avoir commis un crime dans la certitude qu'il était nécessaire, ne la laisse jamais en place, mais mobilise le mouvement, toujours prête à fuir: "Sans cesse et de partout, elle se sent chassée» $(23,26)$, ce qui l'assimile, d'une certaine manière, à « ceux de l'exil » comme les Russes (45), ou les Juifs (25).

Il s'agit ici d'une sorte de fable, celle qui illustre l'état de conscience aiguë de la responsabilité et le questionnement sur les tenants et aboutissants du devoir envers soi-même et envers autrui. Jusqu'où suis-je responsable, susceptible de devoir rendre des comptes? Quelle est ma place dans ce bilan? À partir de quand suis-je condamnable? Et en fonction de quels critères?

Louise, locatrice d'un cabanon en bord de mer en Équateur, est chargée de garder des abalones, sortes de coquillages. Mais ce travail ne l'amuse pas, devient un poids et, lasse, elle les néglige... ils finissent par pourrir. Elle trahit ainsi la promesse faite au propriétaire de veiller à ces biens. Pire, elle y boute le feu, dégoûtée par leur masse informe, un événement, contre toute attente, désiré par les habitants du coin qui se prêtent à ce sacrifice et l'aident à incendier l'océan, en y répandant du pétrole, pour une fête apocalyptique. Ce crime n'en serait pas un s'il ne renvoyait à 
l'écœurement de Louise enfant devant un adulte qui, en sa présence, avait fait souffrir une limace en la brûlant de sa cigarette. Ce crime commis en Équateur la poursuit jusqu'au Mexique où elle se croit surveillée. De fait, il devient évident que ce ravage est connu de tous. La prise de conscience de sa propre cruauté lui donne la mesure des torts qu'elle est capable de déclencher. Mais, tout aussi paradoxalement, ce crime est vu comme nécessaire : "Comme si elle avait rempli son devoir. Comme si elle avait accompli ce qu'on exigeait d'elle » (17); «En réalité, elle aussi se revoit en Équateur. Au bord de l'Océan. Quelques minutes avant que l'idée du crime ne jaillisse en elle, avec son côté impérieux de l'acte nécessaire » (47). L'être humain est pris dans l'engrenage du meurtre et Louise en est consciente au plus haut point. Mais quelle est l'importance et le poids de coquillages brûlés, de l'océan incendié? Le propriétaire n'était-il pas lui-même un «vieux bandit », aux «petits yeux cruels » (83)? La futilité du délit face à l'ampleur du questionnement moral de Louise, à sa culpabilité mesurable à la paranoïa qu'elle développe, sa certitude d'avoir commis un méfait condamnable, tout cela place le lecteur face à ses propres responsabilités. Jusqu'où suis-je responsable? Ne sommes-nous pas tous coupables? Ne devrions-nous pas, comme Louise, sonder notre responsabilité - et par là forcément nous responsabiliser? Les désajustements du monde, son impitoyable cruauté, Louise les prend en charge en une sensibilité poreuse, incapable de distinguer le dedans du dehors : «Elle doit trahir le malaise qu'elle ressent; à penser à son crime, à observer les putos ${ }^{1}$, elle a l'impression que tout se confond dans sa tête, qu'il existe une similitude entre ces deux scènes » (18).

Crime et anéantissement de soi vont de pair. Par son crime, Louise s'annule. Un contexte, un passé, une histoire sont effacés, brutalement détruits, en une table rase invitant à la fuite. Cette décision d'un changement radical est le prolégomène nécessaire à la nouveauté, à l'accueil de l'inouï. La destruction semble ici la condition d'une renaissance : "L'anéantissement dont elle se croit avide n'est pas la mort. Plutôt un bouleversement total qui permette un renouveau » (54). Car, chez elle, ce crime est lié à la passion. Une passion sans doute condamnable, elle aussi, puisque Louise aime "quelqu'un qui était né du "mauvais côté" » (38), le fils d'un général sous Vichy : "Pour des raisons bien différentes, elle se savait, elle aussi, née du mauvais côté. Jamais, jusqu'au jour du crime, elle ne s'était sentie comme les autres. Elle était stupéfaite de voir comme les hommes ligotaient leur propre vie » $(I d$.$) . Et les paradoxes se poursuivent chez ces amants : F. et elles ne se$ ressemblent pas : «Louise aimait F. parce qu'il était exactement ce qu'elle n'était pas » (68); «Qui sait si F., lui aussi, ne m’a pas aimée parce que j'étais tout ce qu'il n'était pas? » (69). Le geste incendiaire de Louise n'est pas avoué à F., mais la passion les soude «à l'ombre du crime » (62); ils sont liés comme si le crime de Louise était la condition sous-jacente à cette passion. En réalité, ces deux événements se rapprochent par leur caractère d'exception, leur

${ }^{1}$ Les "putos", forme masculine de "putas", désigne les jeunes garçons qui se prostituent. 
radicalité, leur capacité à exclure Louise de tout ce qui ne renvoie pas à ce qu'ils ont d'extraordinaire : "Peut-être, pense Louise, mon amour pour F. m'exclut-il de tout ce qui n'est pas lui, comme mon crime m'a exclue de tout ce qui n'était pas dans l'ordre de cet acte » (23). Crime et passion se renvoient en miroir, poreux l'un à l'autre : «l'amour lui a mis un goût de mort sur les lèvres » (23). Leur interdépendance trouve sa synthèse, d'abord à travers l'image de la corrida "dans son large mouvement de passion et de mort» (56), ensuite dans le recueil de poésie de Jean Paul Guibbert, Alyscamps - le titre renvoie au site funéraire romain -, où mort et vie se nouent en une fluidité mélancolique ${ }^{2}$ (cet ouvrage, viatique pour Louise, lui fait comprendre que « seul ce qui était fracassé durait» [64]). Enfin, ces deux engagements, le premier, le crime - incendier en une impérative nécessité ce qui s'impose à soi contre sa volonté, soit dire non - et le deuxième, la passion, sont les actes posés pour amener à la résilience « certaines images où la rage le dispute à la terreur » (84) :

Elle oubliera que ses désirs n'avaient pas de corps. Elle oubliera que la petite fille qui portait son nom avait été violée. Elle ne saura plus quand cela s'est passé. Elle ne se demandera plus pourquoi elle n'est pas morte, alors, même si quelque chose en elle a péri à cet instant. (Id.)

L'étincelle qui met le feu à l'Océan et incendie Louise vient donc de l'affrontement des éléments opposés et de l'assomption de leurs contradictions. La littérature garantit la compréhension de l'importance vitale de ces paradoxes à résoudre ou à maintenir tels quels : "Je vois les lettres de F. dans ma valise ouverte, je vois Alyscamps sur le lit, je dis que je m'aime » (73).

\section{La Séduction des hommes tristes : l'amour contre le crime}

Si Le Gardien d'abalones met en scène Louise Keil, seule avec ellemême, dans une cabane en face de la plage en Équateur, La Séduction des bommes tristes reprend, presque trois décennies plus tard, un décor étrangement similaire, celui d'une case isolée sur une colline au Mexique, sous la voûte céleste, au bord de l'Océan pacifique, où deux personnages aux sentiments contradictoires confrontent leurs émotions sur l'échiquier du récit, chaque chapitre se constituant en damier, noir et blanc, dans l'avancée de la résolution de leurs conflits. Il s'agit d'une scène théâtrale annoncée dès l'ouverture ${ }^{3}$, celle de la descente de la maisonnette vers la plage de Pochutla, à la tombée du jour, où s'y joue une réflexion de l'homme face à lui-même dans la rêverie de ses souvenirs, suivie de son affrontement avec autrui dans sa radicale différence. Une scène qui se réitère tout au long du récit et où le rapport à l'autre est appelé à se transformer.

\footnotetext{
2 "Mais plus tu meurs et plus tu vis/Et il me parait que tu gis,/Auprès de mon corps dans la pierre", (Guibbert, "Dialogues", Alyscamps, 51).

3 “tandis que le ciel, comme un rideau de théâtre que l'on tire, s'ouvrirait bientôt sur la voûte étoilée” (Lalande 2010, 7).
} 
On ne connait pas le nom de l'homme, un "vieux promeneur» (Lalande 2010, 9), mais bien celui de ses deux chiens, Sol et Luna, dont il s'entoure dans cette descente renouvelée vers la mer, en compagnie de son âne et d'un oiseau noir, sa famille. Ce sont ces animaux qui lui apportent un réconfort, "quelque chose qui ressemblait à de l'amour » (Id.). Sa compagne, Alegría, le rejoint dans ce rituel du soir, invariablement répété comme une litanie: "Il descendait vers la plage ». Ce lieu est cependant très vite dramatisé comme la scène tragique de leur mise à mort, décidée de part et d'autre, car l'on apprend que l'homme est traversé par " une pensée qui l'effrayait [...] oui, ce soir, il la tuerait» (15), car il avait perçu en elle « une hostilité trahie [...] il avait compris» (20); elle-même était déterminée, au même instant, à l'empoisonner : « c'est qu'elle avait décidé, ce soir-là, de lui servir un joli mélange de sa composition » (17).

Car si le lieu et le rituel du soir les unissent, leurs dissemblances sont faites pour les séparer et créer entre eux méfiance et haine : ne sont-ils pas un homme et une femme, « un seigneur et sa concubine, un gringo et son Indienne » (24), avec les différences culturelles, sociales et religieuses comme facteurs de dissension et d'éloignement? Car à ce jeu de face à face se superposent en poupée gigogne phantasmes et projections de soi qui interfèrent et biaisent leur relation au point de ne plus percevoir ni en l'autre ni en soi l'authenticité et l'unicité de l'être.

Lui, vient de ces contrées lointaines européennes, «héritier d'une famille qui avait traversé le $\mathrm{XX}^{\mathrm{e}}$ siècle du côté des révolutions, des émigrations dangereuses [...], il avait élu comme modèle un ancêtre originaire d'Odessa » (37). À l'épopée familiale, magnifiée par le mythe d'une origine grandiose aux fortunes dilapidées, se superpose l'Histoire occidentale que l'homme incarne, celle de l'ancien conquistador, le symbole du maittre et surtout, au Mexique, celle de l'empereur Maximilien qui pensait arriver en terre conquise et qui prit lui aussi une Indienne pour amante, «la India Bonita» (25), à l'insu de Charlotte de Belgique qui, pressentant le désastre, retourna en Europe pour obtenir du soutien auprès des princes et tenter de sauver l'Empire. Cette aventure que retiennent les livres d'Histoire, l'homme la connaissait, car il avait été, dans son enfance, fasciné par le tableau de l'exécution de Maximilien peint par Manet, qu'il avait contemplé au Musée d'Orsay, et il avait, enfant, assimilé les éclairs de la fusillade à ses bougies d'anniversaire : « il avait éteint les flammes, qui, dans son esprit, rappelaient celles jaillissant des fusils pointés sur l'empereur » (27). Comme l'empereur, l'homme avait accepté «la India Bonita qui s'était glissée dans son lit », car elle était le contraire de «celle qui avait massacré sa vie » (31). Alegría, sa compagne, est ainsi assimilée à la maitresse de l'empereur, en une sorte de superposition de leur vie et de leur destin : «ses pensées glissèrent vers l'empereur Maximilien qui avait eu, comme lui, un enfant de sa maittresse, il ressentait une sorte de compassion fraternelle pour cet homme abandonné 
de tous» (85). À l'instar de l'empereur, lui non plus «n'avait pas été davantage accepté par les Mexicains » (26) et, malgré les avertissements de ses parents, de l'oiseau noir, malgré les signes avant-coureurs prémonitoires, le sort réservé à l'homme rejoint le destin funeste de l'empereur.

Elle, se situe du côté des pauvres, des vaincus, du peuple arrimé à sa survie, dans la soumission et la haine de ce qui leur fait courber l'échine. Elle vient à l'homme par calcul et manipulation afin d'améliorer un tant soit peu sa situation, même si c'est pour trahir les siens : elle vit avec un blanc tout en le méprisant, car il lui assure une certaine protection, ce qui fait enrager son entourage :

[...] le cœur d'Alegría était rempli de remords et de colère, que peut-on faire d'autre quand on est démuni de tout?, sinon prendre ce qu'on peut prendre [...] elle aimait son ennemi, or, cet homme ignorait qu'il était son ennemi, il croyait en l'amour de l'Indienne, il aurait dû croire aussi en sa haine, mais cela, c'était trop compliqué pour le gringo! (43-44)

Attachée à une série télévisée au scénario sentimental, l'Indienne projette sur son couple les stéréotypes assénés par le culebrón - celui de l'amour impossible entre un homme d'une classe sociale élevée et d'une fille du peuple à laquelle il s'attache superficiellement, puis qu'il abandonne. L'aventure de l'héroïne, c'est la sienne! Et les épisodes commandent ses variations d'humeur. Sa propre histoire s'emboîte en abîme dans le romanfleuve au point que, dans un quiproquo où elle tempête contre l'abandon de la malheureuse amante enceinte par le bel acteur, le vieil homme croit qu'elle parle d'elle, et, contre toute attente, à rebours de la série télévisée, lui témoigne une joie et une reconnaissance mêlées d'amour et de tendresse.

La réalité est donc différente des séries. Les fantasmes amoureux d'Alegría pour l'acteur sont soudain perturbés : «la désespérait l'étrange et nouvelle tendresse de son gringo pour elle » (79); dans un processus de désaliénation, elle en vient à prendre conscience de l'être qu'elle a en face d'elle : «mais voici qu'elle regardait l'étranger et qu'elle éprouvait quelque chose qui n'avait plus rien à voir avec l'intérêt, la manipulation, le mensonge, mais qui se rapprochait de la pitié, et même d'un étonnant sentiment maternel envers cet homme âgé [...] il avait besoin d'elle» (130). Alors qu'il est cerné sur la plage par des bandits décidés à les tuer, Alegría montre de l'aplomb et ramène son compagnon et ses animaux vers la maison, consciente que leur fin à tous est proche, mais l'amour lui a enlevé toute peur: «personne ne s'y trompa, c'était l'amour qui avait transformé sa voix, un amour qui éloignait la peur» (132).

Sur la plage, sous la voûte céleste, s'affrontent le blanc et le noir. Sol, le chien, veille à la sérénité des lieux : " prêt à dénoncer par ses aboiements le bruit différent, donc inquiétant» (8). Les ténèbres ne sont jamais totales, elles sont dissipées par « la lune et les premières étoiles [que l'on voit] briller fort dans la conque du monde » (33). L'homme s'y découvre heureux : «la 
lune brillait fort au-dessus de la mer et la plage en était tout illuminée. On voit comme en plein jour!» (77). La lumière perce les ténèbres et finit par triompher : «Malgré la lumière qui inondait à présent la plage, Alegría avait la sensation de courir dans l'obscurité » (130); associée d'abord à la bande de voyous, elle se détache en fin de roman de ce "peuple d'ombres» (138) dans une transmutation qui la pousse à rejoindre l'amour qu'elle vient de découvrir pour le vieil homme et affronter avec lui la mort.

\section{Nous veillerons ensemble sur le sommeil des hommes: l'amour est plus fort que la mort}

Ce roman de Françoise Lalande met en scène trois membres de la diaspora de la famille Keil, la première, Léa Keil, est née à Berlin, la seconde, Lila, provient des Ardennes belges et le troisième, Julius, de Seattle, aux É.U.. Tous trois se retrouvent en fin de roman à Tel-Aviv pour une réunion familiale des membres dispersés dans le monde. Ils décident qu'ils ont atteint un degré de bonheur tel, celui de se connaître et reconnaitre, qu'il n'est pas indispensable de prolonger leur vie au-delà, et ils se donnent ensemble une mort douce. Le bonheur d'aimer se prolonge ainsi dans la mort. L'ensemble du roman montre en ce sens une résolution de l'ensemble des paradoxes de l'existence. Depuis les conflits intérieurs, toujours un peu en porte-à-faux, de Louise Keil, dans le premier roman, on aboutit ici à une intégration des contradictions, acceptées dans la sérénité, chez les principaux protagonistes.

\section{a. Berlin. Histoire de Léa Keil}

En premier lieu, la narration se construit sur des renversements de situation. Petite fille juive, Léa est cachée avec sa mère, Myriam-la-Jeune, par deux Allemands dans leur cave à Berlin durant la guerre et, malgré la précarité de cette existence et de ce lieu, sa mère lui donne une confiance inébranlable en la vie. À la fin de la guerre, c'est Myriam-la-Jeune qui, à son tour, doit sauver ses protecteurs au moment de l'arrivée de l'armée russe. Elle rencontre l'amour dans les ruines de cette ville, mais aussi la mort, à la suite d'une chute lors de sa reconstruction : «Mon squelette ruiné est à l'image de cette ville» (Lalande 2012, 30). À l'orphelinat des enfants juifs, Léa développe son génie du violoncelle et devient une artiste au talent exceptionnel, avec l'appui logistique de son compagnon d'enfance, David, son frère symbolique, juif lui aussi. Un homme, Lucas Horn, assiste à deux de ses concerts à Berlin. Pensant la reconnaître, il l'attend à la sortie des artistes et ils échangent un regard. Peu après, il est agressé. Léa l'apprend par les journaux et vient voir dans le coma cet homme qu'elle désire. Elle ne se fait cependant pas connaitre, voulant perfectionner son art et mûrir avant de l'aimer. Au faite de sa gloire, elle décide de ne plus jouer que dans des lieux et pour des gens d'exception. David entame alors en secret la recherche de Lucas Horn, alors que Léa le retrouve au pied de la chute du mur de Berlin, symbole de l'abolition de leur séparation et de l'avènement de leur fusion 
rendue possible. Le passé rattrape cependant les deux amants : Lucas a été momentanément embrigadé dans les jeunesses nazies; Léa découvre cependant qu'elle continue à l'aimer et lui montre le visage de sa mère qui n'est autre que le premier amour de Lucas. Il n'en dit rien, bouleversé, mais ce secret inavoué fissure leur amour.

Ceux qui ne peuvent résoudre les contradictions à travers l'amour sont voués à la mort : la mère de Léa, Myriam-la-Jeune, était devenue « une femme sans pardon [...] qui brûlait de haine » (18) et qui en elle «lovait le secret désir d'en finir avec soi-même » (29), car cette haine est d'abord une haine de soi (« quelle haine de soi? serait-ce la clé de l'humanité? » (33)), alors que le sentiment amoureux pour le jeune inconnu contaminait l'environnement désolé, celui des ruines «constituant le paysage de sa résurrection» (32), un lit de pierre sur lequel elle faisait l'amour et qui annonçait un nouveau départ. Mais en elle, le constat est cependant celui de l'effritement; elle tombe ainsi d'une façade en reconstruction à l'instar d'un ancêtre, tombé des années auparavant non loin de là du toit d'une synagogue. D’une certaine manière, Myriam-la-Jeune reproduit les échecs familiaux. Lucas Horn, de son côté, cesse de se construire dans sa relation à Léa, car il ne peut se dégager du trouble causé par la découverte de sa passion à la fois pour Myriam-la-Jeune et pour sa fille Léa.

Le texte travaille, en second lieu, les contradictions inhérentes à l'humain. Léa les intègre joyeusement, car elle pourfend de façon naturelle les interdits liés à l'esprit dualiste. En cela, elle sort de ce qui stérilise la pensée rationnelle pour ouvrir l'humanité à d'autres horizons et vient toucher des besoins enfouis dans le cœur des hommes. Léa est une " orpheline radieuse » (50); elle a « le poignet solide malgré la finesse, une volonté de fer malgré la soie du corps » (40); son art comme son être confère cet entre-deux, cette rencontre des contradictions, pointe ce lieu où elles se tiennent ensemble sans se confondre en une synthèse éblouissante: "dès le premier coup d'archet de la violoncelliste, il a ressenti un coup de lame dans son ventre, et ce coup était bon » (41). Cet art de l'assomption des contradictions, de leur accueil, en soi, se communique donc à autrui - plus précisément ici à Lucas Horn - sous la forme d'un «bonheur douloureux » (42). Car Léa semble initiée à la connaissance de soi; elle se sait à la fois dans la joie et la cruauté; tuer un insecte «l'excite à ce point, la rend totalement méchante » (68), car la joie n'est pas à confondre avec la bonté. Mais, se connaissant, ce qu'elle offre à autrui est « sa part de lumière, sa jumelle solaire, et [elle] garde en elle sa part d'ombre, des sentiments furieux » (59). Sa création est le résultat de la transformation «de ses épreuves en joie » (58), ce qui l'anime à jouer est « une volonté d'amour » (63). Avoir frôlé des gouffres confère à la vie cette densité de la jouissance, nous gonfle de cette joie-là, annule les peurs paralysantes, dont celle de la mort. La joie de Léa semble venir d'un autre monde et donne la sensation « d'être privilégié, celle de respirer dans un lieu nouveau, de vivre en fraternité avec les autres, hors du monde profane » (45), 
peut-être est-elle même propice à une transformation des êtres et des choses, à travers « force, beauté et sagesse humaines ", même si Léa sait que cette sérénité arrachée à la folie des hommes ne durera que le temps du récital, et que, celui-ci terminé, il ne faudra pas attendre longtemps pour que les hommes renouent avec les égoïsmes et les injustices [mais aussi] que la paix se fera quasi malgré eux (car l'homme contemple dans le miroir son pire ennemi) (72).

Cette joie intrinsèque est portée chez les protagonistes par la force et la générosité que confère la maturité (93).

Le lieu de réconciliation des éléments contraires chez Léa est le centre du désir, d'où jaillit l'illumination des corps et des esprits. L'éclat de ce désir atteint toute sa magnificence dans la relation amoureuse, la rencontre érotique d'un amour solaire qui vous met des "étoiles dans tout le corps » en un feu d'artifice (109). Ce désir éblouissant de Léa brise toute frontière, entre son corps et ceux d'autrui, anéantit les nombreux interdits liés à la dualité qu'elle transgresse en multipliant ses amours, à deux, mais aussi à trois (" elle décide de conclure la nuit en compagnie de David et de l'organisateur, Elie Nacar, panier où de jeunes chiens se mordillent et s'exténuent, avant de tomber endormis, corps enchevêtrés » [73]), et à mille : son violoncelle est ainsi le vecteur de son désir érotique qui électrise un public invité à se joindre à cette relation amoureuse qui humanise les êtres : « elle parle d'amour par la musique, de la délivrance des cœurs par la voix humaine du violoncelle, de l'émotion érotique des corps que celui-là provoque, ils écoutent, adhèrent au bonheur humain qui fait reculer la barbarie » (79).

\section{b. Petit royaume et grande république. Histoire de Lila Keil}

Lila Keil est née pendant la guerre dans une famille «handicapée de l'amour » (133), à la «terrible incapacité d'amour, une entrave ancestrale » (222), au sein de laquelle les «bombes intimes, avec leur déflagration invisible aux yeux des autres» sont elles aussi destructrices (128), où les mariages prennent l'allure d'enterrement (132) et où ceux qui sont revenus des camps «n'en étaient jamais revenus» (142). Dans cet univers aux paradoxes sans échappatoire, où chacun se retrouve pris au piège d'un monde sans joie et d'un manque à vivre, Lila se construit tant bien que mal « contre » sa famille : « elle n'aime ni sa mère ni son beau-père » (127). Très tôt cependant, elle pressent qu'une conversion de ces ténèbres en lumière est possible, «captant d'emblée ce qui fait vivre, c'est-à-dire l'amour [...] elle changera l'attirance pour les gouffres en désir de vivre intensément » (129). Le processus de construction qu'elle entame inclut ainsi « le courage de tuer ce qui vous détruit » (156). Dans le monde de violence - politique, familiale, intérieure - de son enfance, Lila apprend à discerner le blanc du noir à partir de la différence avérée entre le jour et la nuit. Car si le jour la ferre d'impressions négatives, la nuit est porteuse de réparations. Elle décide ainsi que « le réel n'existe pas, seul importait ce que les hommes en racontaient » 
(139). Lila entra ainsi en fiction (Id.), «la fiction était son pays » (144). À partir de ces distinctions entre jour et nuit, réel et fiction, Léa mena deux vies parallèles et opposées, la littérature étant le vecteur permettant la résolution des contradictions : «Lila s'emparait des désastres humains et les mettait en fiction» (139).

Cette capacité de tenir ensemble, sans les confondre, douleur et joie, pour juguler l'une par l'autre et atteindre la sérénité est la lutte sans répit de toute une vie. Car si elle s'exerce tôt, cette métamorphose n'épuise le potentiel de violence de départ qu'en fin de course. Profondément ancrée dans l'inconscient, cet héritage autodestructeur se manifeste dans la prime enfance de Lila Keil par son désir d'une vie monastique où tourmenter sa chair (143), puis dans sa fascination à l'adolescence d'un «tyran violent», le premier amoureux jaloux - celui d'une longue série - dont Lila admira la fureur, subissant humiliations et agressions (146) jusqu'à ce que cette violence soit maitrisée mentalement et physiquement, au seuil de la maturité, dans un renversement où Lila devient catcheuse, s'exerçant de jour dans les salles de sport, pour affronter la nuit ses propres pulsions destructrices, soit en public - sur un ring -, soit en privé, en une lutte érotique avec son adversaire. Cette extériorisation fictive de la violence est à ses yeux « la bonne réponse à la violence réelle » (170). Portée par sa propre rage, Achiléa, Dora ou Gladiatora gagne tous les combats - écrivaine de jour, lutteuse de nuit , dans un face à face de ses propres contradictions - «elle le déteste, elle l'aime » (199) - qui n'est qu'un combat avec elle-même, jusqu'à ce que s'épuise sa propre haine : "Lila s'est délestée de ce qui était mauvais en elle et qui agressait le monde » (241). La littérature est bien un sport de combat qui engage « une descente dans les profondeurs humaines » (149) et à travers laquelle " la découverte de ses origines, au lieu de l'empoisonner, l'a libérée » (245). L'absence de peur et une douceur, inconnues jusqu'alors, signent l'accomplissement de sa métamorphose.

\section{c. Seattle. Histoire de Julius Keil}

Julius Keil affronte ses relations familiales par le prisme d'une mère aux oppositions et partis pris contradictoires, chronologiquement instables et sujets à renversements, "une ogresse névrosée » (260). L'inversion des rôles genrés avait touché son père et sa mère : « on comprenait que l'homme du couple, c'était elle, tandis que lui, Albert, laissait s'épanouir sa part féminine » (255). À son décès, Dahlia comprend cependant que son mari a aimé une autre femme, ce qui déchaîne sa furie contre l'ensemble de la gent masculine, y compris son fils avec lequel elle entretient dorénavant une relation en tension, entre amour et haine ("Julius n'était plus que faiblesse, tandis que sa mère devenait plus dure chaque jour » [259]), faite d'opposition contre son épouse Deborah (" immédiatement, Dahlia détesta cette femme » [268]), puis d'alliance avec elle quand elle comprend que son fils, marin, voit lui aussi une maîtresse au port. Les contrastes s'accumulent : Julius, « beau 
comme un ange », aime "une fille au visage de cigale [et] certains sourirent devant le nouveau couple si mal assorti, un homme d'une beauté troublante et un laideron » (277), un contraste extérieur, mais aussi intérieur, car Rachel "si sombre, semblait avoir un besoin éperdu de cet homme de lumière " (288). Dahlia cependant se rapproche de Rachel lorsqu'elle apprend que cette dernière est enceinte, mais l'infidélité que Deborah avait acceptée de son mari, elle ne la supporte pas chez sa belle-mère. C'en est trop! Elle divorce. C'est alors que Rachel, le « petit mec » de Julius (315), apporte son nouveau-né à Deborah pour disparaittre ensuite dans la nature; cette dernière accueille l'enfant avec l'amour inconditionnel qu'elle incarne. Il faudra un renversement de perspective radical pour que Julius se rende compte de sa véritable nature. Celui-ci est symbolisé dans le roman par le vertige qui le prend en marchant sur la terre ferme. Ce mal de terre où le sol tangue le conduit dans les bras d'un autre marin : «Julius Keil, l'ange fatigué d'être un ange, se transformait, amenant plus d'obscurité pour les siens que de la lumière, il était temps de découvrir qui il était» (313). Car la paix dans le monde doit d'abord provenir d'une paix intérieure : «il pensa que le monde ne serait jamais en paix, il pensait cela parce qu'il n'y avait encore rien d'apaisé en lui » (325). Julius découvre alors sa nature gay et se décide à parcourir le monde, comme si se trouver produisait le mouvement. Les voyages lui dévoilent une autre composante de sa personnalité, celle de Juif américain. C'est cette connaissance de soi, de ces appartenances multiples, reconnues et assumées, qui le conduisent en bout de course à retrouver une unité avec les siens, par-delà les antagonismes qui avaient pu se créer. Il découvre également au cœur de son milieu d'origine la bonté du monde dans la fraternité des amis de son père qui avaient secrètement veillé sur lui tout au long de sa vie :

[...] il s'était sans doute parfois cru seul au monde, alors que des hommes veillaient sur lui, chacun avait assisté à ses amours sans se permettre de le juger, seul le désir de lui porter secours si le besoin s'en était fait sentir guidait les amis de son père, Tu étais seul, et tu n'étais pas seul, Julius, comme chacun d'entre nous. (343)

\section{d. Israël. Réunion de la famille Keil}

Dans cette ville paradoxale qu'est Jérusalem, «lieu de culte et de déchirure, là où, dans un même mouvement, les hommes prient pour leur salut et le perdent» (377), se retrouvent Léa, Lila et Julius Keil pour une réunion de toutes les «branches d'un acacia » que représente l'ensemble de la famille Keil. Léa, forte de l'amour de sa mère et de la joie qu'elle lui a léguée (393), qui assume sa contradiction d'avoir aimé un Allemand, elle, la Juive cachée de Berlin; Lila, qui a subsumé toutes les apories de la mentalité Keil, car elle a hérité du bonheur de vivre de sa grand-mère Liza; et enfin Julius qui savoure son intégrité. Tous trois se reconnaissent dans un sentiment rare partagé, " parfois délicat à cerner, souvent peu décrit et qui se nomme "loyauté" (376), mais aussi une "solidarité [qui] se tissait entre eux [...] une fusion plus vive qu'une fusion amoureuse" (381). Ensemble, ils constatent les liens qui se tissent entre la Palestine et Israël par des hommes 
de bien - surtout des poètes et des médecins -, par-delà les murs; mais audelà de cette vision des échanges fraternels possibles entre les deux pays, ils éprouvent surtout entre eux cette unité qui soude et dégage de tout conflit; hors contradiction, "ils forment un trio d'amants, calmes, forts, à l'image de ceux qui ont vaincu la barbarie" (391). Léa offre à sa famille retrouvée sa dernière composition dans les jardins de Yad Vashem : "L'Amour du monde a été dit dans la forêt des Justes, en cette nuit noire, l'amour a vaincu la haine, le Haïm!" (394). Cet amour qui les unit est une forme d'accomplissement, un degré de perfection et de bonheur qui ne peut ou n'a pas à se prolonger. Les trois amants se suicident ensemble, car ils "ont changé la malédiction familiale en joie" (425). La mort n'est pas effrayante, mais douce, aussi douce que la vie découverte à travers cette joie.

\section{Conclusion}

Louise Keil, dans Le Gardien d'abalones ne se déprend pas de la culpabilité d'avoir dit non à une situation qui ne lui convenait pas et de l'avoir quittée dans la violence. Bien qu'elle reconnaisse l'aspect salutaire de son crime, le remords la rattrape dans l'image de l'Océan pacifique submergeant la ville et se vengeant ainsi d'elle dans un tsunami qui l'engloutit. Les paradoxes se succèdent dans ce roman où la passion ne peut fleurir qu'à l'ombre d'un crime, celui d'exister dans l'affirmation de soi face à autrui. La Séduction des hommes tristes reprend la même scène théâtrale d'une cabane en bord de plage, mais les contradictions s'y affrontent cette fois à travers deux personnages divisés par le mépris de leurs différences sociales et culturelles. Leur mise à nu, hors phantasmes et dans le dépouillement de leurs a priori, les unit dans l'amour, malgré la mort qui les attend. Cette contradiction, la plus insoluble de toutes, celle de la vie et de la mort, se résout dans le dernier roman, Nous veillerons ensemble sur le sommeil des morts, où les trois personnages s'aiment jusque dans la mort. Symboliquement, la triangulation résout les conflits inhérents au dualisme où s'opposent de manière insoluble les éléments contradictoires. 


\section{Bibliographie}

Guibbert, Jean Paul. La Chair du monde. Poèmes 1962-1982. Paris : Éditions Phébus, 1987.

Lalande, Françoise. Le Gardien d'abalones [1983]. Bruxelles : Labor, 1994.

---. La Séduction des hommes tristes. Avin : Luce Wilquin, 2010.

---. Nous veillerons ensemble sur le sommeil des hommes. Avin : Luce Wilquin, 2012. 